\title{
Clinical and Radiographic Comparisons of Distal Tibia Shaft Fractures Stabilized with an Intramedullary Nail: Blocking Pin Versus Blocking Screw
}

Haotian Wu ( $\nabla$ haotian.zj.wu@hotmail.com )

Hebei Medical University Third Affiliated Hospital

Xiaodong Bai

Hebei Medical University Third Affiliated Hospital

Zhaohui Song

Hebei Medical University Third Affiliated Hospital

\section{Shuangquan Yao}

Hebei Medical University Third Affiliated Hospital

Song Liu

Hebei Medical University Third Affiliated Hospital

Lijie Ma

Hebei Medical University Third Affiliated Hospital

Pengcheng Ren

Hebei Medical University Third Affiliated Hospital

Pengcheng Wang

Hebei Medical University Third Affiliated Hospital

Research article

Keywords: Distal tibia shaft fracture, Blocking pin, Blocking screw, Intramedullary nail

Posted Date: June 23rd, 2020

DOI: https://doi.org/10.21203/rs.3.rs-37184/v1

License: (c) (1) This work is licensed under a Creative Commons Attribution 4.0 International License. Read Full License 


\section{Abstract}

Background: Distal tibia shaft fractures have a high risk of mal-alignment when treated with intramedullary nailing (IMN). Permanent blocking screws (BSs) and temporary blocking pins (BPs) are often used as supplements to help IMN get good alignment. The purpose of this study was to compare the clinical and radiographic outcomes of temporary BPs and permanent BSs for distal tibia shaft fractures.

Methods: From March 2014 to May 2019, a total of 89 patients with distal tibia shaft fractures were included in this retrospective study and divided into two groups. All fractures were located below the isthmus but $4 \mathrm{~cm}$ above the tibial plafond. The differences in operating time, intraoperative bleeding, fibula plate fixation, number of BP/BS, initial and final alignment, loss of reduction, fracture healing time, and postoperative complications were analyzed. Functional outcomes of the ankle were assessed with the American Orthopedic Foot and Ankle Society (AOFAS) score.

Results: All patients underwent a minimum follow-up of 13 months. The operating time was $(73.9 \pm 6.1)$ min and $(80.2 \pm 8.6) \mathrm{min}$ in the BP group and BS group, respectively $(P<0.05)$; intraoperative bleeding was $(88.2 \pm 18.0) \mathrm{mL}$ and $(92.9 \pm 26.6) \mathrm{mL}$, respectively $(P>0.05)$; initial reduction deformity in coronal plane was $(0.5 \pm 2.3)$ degrees and $(0.9 \pm 2.3)$ degrees, respectively $(P>0.05)$; final alignment deformity in coronal plane was $(1.2 \pm 2.7)$ degrees and $(1.3 \pm 2.4)$ degrees, respectively $(P>0.05)$; initial reduction deformity in sagittal plane was $(0.6 \pm 2.2)$ degrees and $(0.6 \pm 2.4)$ degrees, respectively $(P>0.05)$; final alignment deformity in sagittal plane was $(0.9 \pm 2.3)$ degrees and $(1.0 \pm 2.2)$ degrees, respectively $(P>0.05)$; As for the loss of reduction, there was no significant difference in sagittal plane between the two groups $(P>0.05)$. Although the loss of reduction in the coronal plane of the BP group (0.8 \pm 0.7 degrees) is statistically higher than that of the BS group $(0.5 \pm 0.5$ degrees), the small difference was not clinically important $(P<$ $0.05)$. No significant differences were found between the two groups in fracture healing time, AOFAS score and complications $(P>0.05)$.

Conclusion: Both temporary BP and permanent BS can help achieve equivalent overall alignment. However, BP technique is simpler and takes less time.

\section{Introduction}

Tibial fractures can successfully be treated with intramedullary nailing (IMN) [1-3]. However, distal tibiashaft fractures are challenging as the expanding diaphysis going to the metaphysis increases the size of the intramedullary canal [4]. The reported incidence of mal-alignment ranges from $10-29 \%$ [5-7].

The blocking screw (BS), introduced by Kretek et al [8,9], can narrow the medullary cavity and direct IMN to a desired position, which has become a popular surgical procedure for nailing a distal tibia shaft fracture. However, it has potential complications such as screw breakage, iatrogenic fracture, soft tissue irritation, and hardware removal [10-14]. 
To overcome these issues, Shahulhameed et al.[12] proposed to use a smooth and elastic Steinmann pin to facilitate fracture reduction, and finally replace it with a permanent BS. With the development of modern IMN, Wang et al.[15] and Poyanlı et al.[16] found that when the metaphyseal fracture was reduced with a temporary Kirschner wire, the fracture reduction could be well maintained even if the Kirschner wire was removed directly rather than replaced with a permanent BS. This new technique can reduce the operating time and avoid permanent soft tissue irritation. However, the main concern about it is that there is a potential decline in construct stiffness without the permanent BS. To date, there is no large sample clinical study comparing the efficacy of temporary blocking pin (BP) and permanent BS for nailing a distal tibia shaft fracture.

In our institution, the BP or BS has always been used as a supplement when acute distal tibia shaft fractures were treated with IMN. The purpose of the present retrospective study was to compare the clinical and radiographic outcomes of BP and BS.

\section{Materials And Methods}

\section{Patients}

Every skeletally mature patient with an acute fracture of the distal tibia shaft who underwent IMN fixation with temporary BP or permanent BS at our institution between March 2014 and May 2019 were selected for this retrospective study. Exclusion criteria included AO/OTA 43 fractures, open fractures, pathological fractures, multiple fractures, preexisting diseases affecting activities of the ipsilateral lower limbs, and insufficient radiographic or chart data. All fractures were located below the isthmus but $4 \mathrm{~cm}$ above the tibial plafond. The clinical study was approved by the Ethics Committee of the Third Hospital of Hebei Medical University in China, and written informed consents were obtained from all participants.

A total of 89 patients were identified. There were 56 males and 33 females, with a mean age of 43.7 years. The mean distance from the plafond was $8.76 \mathrm{~cm}$ (Table 1). Based on whether BP or BS was used during nailing, 46 patients were divided into the BP group and 43 into the BS group. There were no significant differences in baseline demographic characteristics, including age, gender, injury mechanism, AO/OTA classification, distance from the tibial plafond, interval from initial injury to surgery, and followup time (Table 1). 
Table 1

Patients' baseline demographic characteristics

\begin{tabular}{|c|c|c|c|}
\hline Parameters & BP Group $(n=46)$ & BS Group $(n=43)$ & P-value \\
\hline Age (year, mean \pm SD) & $42.3 \pm 9.1$ & $45.1 \pm 10.5$ & 0.178 \\
\hline Gender [ n (\%)] & & & 0.367 \\
\hline Male & $31(67.4 \%)$ & $25(58.1 \%)$ & \\
\hline Female & $15(32.6 \%)$ & $18(41.9 \%)$ & \\
\hline Side of injuryn [ $\mathrm{n}(\%)]$ & & & 0.753 \\
\hline Left & $24(52.2 \%)$ & $21(48.8 \%)$ & \\
\hline Right & $22(47.8 \%)$ & $22(51.2 \%)$ & \\
\hline Injury mechanis [ $\mathrm{n}(\%)]$ & & & 0.485 \\
\hline Falls from standing & $19(41.3 \%)$ & $15(34.9 \%)$ & \\
\hline Fall from height & $8(17.4 \%)$ & $12(27.9 \%)$ & \\
\hline Industrial injury & $6(13.0 \%)$ & $7(16.3 \%)$ & \\
\hline Traffic acciden & $11(23.9 \%)$ & $9(20.9 \%)$ & \\
\hline Others & $2(4.3 \%)$ & $0(0 \%)$ & \\
\hline OTA/AO classification [ $\mathrm{n}(\%)]$ & & & 0.886 \\
\hline $42 \mathrm{~A}$ & $32(69.6 \%)$ & $28(65.1 \%)$ & \\
\hline $42 B$ & $8(17.4 \%)$ & $8(18.6 \%)$ & \\
\hline $42 \mathrm{C}$ & $6(13.0 \%)$ & $7(16.3 \%)$ & \\
\hline Time to nail (day, mean \pm SD) & $3.2 \pm 1.3$ & $3.6 \pm 1.4$ & 0.121 \\
\hline Distance from tibial plafond $(\mathrm{cm}$, mean $\pm \mathrm{SD})$ & $8.78 \pm 0.86$ & $8.75 \pm 1.20$ & 0.902 \\
\hline Follow-up period (month, mean $\pm S D$ ) & $17.8 \pm 3.8$ & $17.5 \pm 3.1$ & 0.740 \\
\hline
\end{tabular}

\section{Surgical Procedures}

For the BP group, a trans-patellar tendon approach was used with the knee in a flexed position.

Percutaneous manipulation was performed to obtain and maintain an approximate reduction. A 3-mm Steinmann pin was used as a temporary BP. Following the concave side principle [9] or acute angle principle [17], one or more BPs were inserted. The location of the BP is $6.0-7.0 \mathrm{~mm}$ from the center of the canal and $1.0 \mathrm{~cm}$ from the fracture line $[12,17]$. Thereafter, the ball-tipped guidewire was inserted at the 
correct side. Critical techniques included the central placement of the guidewire and reaming to a subchondral level, approximately $5.0 \mathrm{~mm}$ from the distal articular surface [18]. The nail was inserted and locked with two proximal and two distal interlocking screws. Finally, the BP is pulled out directly. For the BS group, the operation procedure was similar to that of the BP group, except that after the nail was locked, the BP was replaced with a 5-mm cortical screw. Special emphasis should be placed on achieving adequate visualization of each process on the distal segments until fixation of distal interlocking screws.

\section{Postoperative management}

Routine antibiotics and anticoagulants were administered as preventive measures. After $24 \mathrm{~h}$ of the operation, patients were advised to begin an early, active, full range of motion of the ankle and knee joints within the limits of their comfort. Gradually, weight-bearing was permitted based on the signs of fracture callus visualized on postoperative radiographs. Radiographs were obtained every month until fracture union and at 12 months follow up. Coronal and sagittal alignments were evaluated using techniques described by Freedman and Johnson [19]. Varus and recurvatum angulation were expressed as negative values, and valgus and antecurvatum as positive values. The radiographs immediately after surgery and 12 months after surgery were used to evaluate the initial reduction and final alignment, respectively, and the angle change between them was used to evaluate the loss of reduction. Mal-alignment was defined as $>5$ degrees in any plane. Fracture union was defined as full weight-bearing without pain and bridging callus of 3 or 4 cortices on x-rays. Failure to achieve this at 6 months and/or by additional procedures undertaken to promote healing was defined as nonunion. Functional outcomes of the ankle were assessed with the American Orthopedic Foot and Ankle Society (AOFAS) score at 12 months follow up.

\section{Statistical analysis}

The data were analyzed with the use of SPSS software 25.0 (SPSS, IBM, Armonk, NY, USA). Continuous data were expressed as mean and standard deviation, and were evaluated using the Student- $t$ test or Mann Whitney $\mathrm{U}$ test based on data distribution. Categorical data were expressed as number and percentage, and were evaluated by Chi-square test or Fisher exact test, as appropriate. $P$ values $<0.05$ were regarded as representing a significant difference.

\section{Results}

All 89 patients were followed up for a minimum of 13 months. As indicated in Table 2, the average operating time of the BP group was significantly shorter than that of the BS group (73.9 vs. 80.2 min, $P<$ 0.001). There was no significant difference in intraoperative bleeding between the BP and BS groups (88.2 vs. $92.9 \mathrm{ml}, P=0.341)$. Six (13.0\%) distal fibula fractures in the BP group were plated, and five $(11.6 \%)$ in the BS group $(P=0.839)$. In the BP group, 39 fractures were reduced with one BP, and 7 fractures with two. In the BS group, 38 fractures were reduced with one BS, and 5 fractures with two. No significant differences were seen in this comparison $(P=0.620)$. The average fracture healing time was 
17.6 weeks in the BP group and 17.4 weeks in the BS group $(P=0.761)$. A total of $5(5.6 \%)$ cases developed fracture nonunion, three $(6.5 \%)$ of which occurred in the BP group, which was not statistically different between the two groups $(P=0.702)$. Two patients $(4.7 \%)$ with nonunion in the BS group were treated with dynamization. Of three patients in the BP group, one was treated with dynamization, and the other two patients healed following a bone grafting procedure. All 5 fractures healed uneventfully. Two patients underwent removal of the permanent BS due to soft tissue irritation( $4.7 \%, P=0.139$ versus $\mathrm{BP})$. No wound complication requiring additional surgical procedures was observed. At last follow-up, the average AOFAS score was 82.4 in the BP group and 84.8 in the BS group $(P=0.148)$. (Table 2)

Table 2

Clinical outcomes

\begin{tabular}{|llll|}
\hline Parameters & BP Group $(\mathbf{n}=46)$ & BS Group $(\mathbf{n}=43)$ & P-value \\
\hline Operating time(min, mean \pm SD ) & $73.9 \pm 6.1$ & $80.2 \pm 8.6$ & $\otimes 0.001$ \\
\hline Intraoperative bleeding (ml, mean \pm SD) & $88.2 \pm 18.0$ & $92.9 \pm 26.6$ & 0.341 \\
\hline Distal fibula plate fixation [n (\%)] & $6(13.0 \%)$ & $5(11.6 \%)$ & 0.839 \\
\hline Number of BP/BS [n (\%)] & & & 0.620 \\
\hline One BP/BS & $39(84.8 \%)$ & $38(88.4 \%)$ & \\
\hline Two BP/BS & $7(15.2 \%)$ & $5(11.6 \%)$ & \\
\hline Fracture healing Time (week, mean \pm SD ) & $17.6 \pm 4.1$ & $17.4 \pm 3.2$ & 0.761 \\
\hline Nonunion [n (\%)] & $3(6.5 \%)$ & $2(4.7 \%)$ & 0.702 \\
\hline Softissue irritation [n (\%)] & $0(0 \%)$ & $2(4.7 \%)$ & 0.139 \\
\hline AOFAS score (mean \pm SD ) & $82.4 \pm 8.4$ & $84.8 \pm 7.5$ & 0.148 \\
\hline
\end{tabular}

The initial and final mal-alignments were detailed in Table 3, and no significant differences were found between the two groups (all $P \llbracket 0.05$ ). As for the loss of reduction, there was no significant difference in the sagittal plane between the two groups $(P=0.251)$. Although the loss of reduction in the coronal plane of the BP group is statistically higher than that of the BS group, this small difference of about 0.3 degrees was not clinically important( 0.8 vs. 0.5 degrees, $P=0.012$ ). At the last follow-up, the mal-alignment greater than 5 degrees was identified in 7 patients (7.9\%), including four patients in the BP group (8.7\%) and three patients in the BS group $(7 \%, P=0.763)$. Six of 7 fractures were initially stabilized in a malaligned position, and the other one underwent a 4-degree loss of reduction during healing. (Table 3 ) 
Table 3

Radiographic alignment

\begin{tabular}{|llll|}
\hline Parameters & BP Group $(n=46)$ & BS Group $(n=43)$ & P-value \\
\hline In Coronal plane (degree, mean \pm SD) & & & \\
\hline Initial reduction deformity & $0.5 \pm 2.3$ & $0.9 \pm 2.3$ & 0.464 \\
\hline Final alignment deformity & $1.2 \pm 2.7$ & $1.3 \pm 2.4$ & 0.849 \\
\hline Loss of reduction & $0.8 \pm 0.7$ & $0.5 \pm 0.5$ & 0.012 \\
\hline In Sagittal plane (degree, mean $\pm S D)$ & & & \\
\hline Initial reduction deformity & $0.6 \pm 2.2$ & $0.6 \pm 2.4$ & 0.918 \\
\hline Final alignment deformity & $0.9 \pm 2.3$ & $1.0 \pm 2.2$ & 0.969 \\
\hline Loss of reduction & $0.5 \pm 0.5$ & $0.6 \pm 0.5$ & 0.251 \\
\hline Initial reduction deformity $>5$ degrees $[\mathrm{n}(\%)]$ & $3(6.5 \%)$ & $3(7.0 \%)$ & 0.932 \\
\hline Final alignment deformity $>5$ degrees $[\mathrm{n}(\%)]$ & $4(8.7 \%)$ & $3(7.0 \%)$ & 0.763 \\
\hline
\end{tabular}

One typical case is presented in Fig. 1.

\section{Discussion}

The current study provides the clinical rationale that the temporary BP and permanent BS can achieve similar clinical and radiographic outcomes in the treatment of distal tibia shaft fractures with IMN. Although the coronal loss of reduction of the BP group is a bit more than that of the BS group, this small difference of about 0.3 degree is not clinically important.

The initial reduction of less than 5 degrees was observed in 83 patients, and 82 patients retained it until the last follow-up. This indicates that a good initial reduction is the key to prevent malunion in the treatment of distal tibia shaft fracture with IMN, which has also been confirmed by other studies. A recent prospective study reported 56 patients with distal tibia shaft fractures fixed with IMNs [7]. 43 patients had good initial alignment ( $<5$ degrees), 42 patients maintained it until the fracture healed, and only 1 case developed from initial good alignment to malunion due to immediate weightbearing against medical advice. A retrospective study by Vallier et al [5] reported 76 patients with distal tibia shaft fractures fixed with IMN. 54 patients had good initial alignment of less than 5 degrees, and all of them maintained it until the fracture healed. Similarly, Guerado et al [14] and Avilucea et al [20] also suggest that a good initial reduction is the key to prevent malunion. However, the initial mal-reduction rate of the current study is significantly lower than the reported rate of $23 \%-29 \%$, which may be related to the fact that the blocking technique can help achieve a more predictable and accurate reduction $[5,7]$. 
The BP and BS achieved similar overall alignment. Whilst there was a statistical difference in loss of reduction in the coronal plane between $B P$ and $B S$ groups $(P=0.012)$, we do not feel that this small difference, of about 0.3 degrees is relevant clinically. The permanent BS may not provide an important clinical improvement in structural stability, which had been confirmed by a recent biomechanical study. Chan et al [21] evaluated the mechanical stiffness of the reamed IMN with two distal 5-mm interlocking screws augmented with or without a medial BS in the treatment of distal tibia fractures. They concluded that there was no significant difference in mechanical stiffness with or without the BS, and the IMN fixation alone can provide sufficient stability to allow for immediate weight-bearing. Moreover, De Giacomo and Tornetta [22] retrospectively analyzed 132 distal tibia fractures fixed with an IMN and concluded that IMN with two distal interlocking afford adequate stability to hold the reduction during union. In their study, no significant loss of reduction (average change of 0.9 degrees in both the coronal and sagittal planes) was observed after surgery. However, in an early reported study, Krettek et al [8] reported that two BSs placed medial and lateral to an 8-mm IMN increased bending stiffness by $57 \%$ compared with three interlocking screws without BSs. But the small diameter unreamed IMN and $3.9 \mathrm{~mm}$ interlocking screws they used were rarely used today, which may generate heterogeneity.

Previous literature suggested using permanent $B S$ as a supplement to IMN $[9,11,23,24]$. However, it must be noticed that our and their studies differed in several aspects. First, their patient group included a large number of OTA 43 fractures and fracture nonunions. In the present study, we only selected acute, closed fractures of the distal tibia shaft (OTA 42), which were more stable and easier to heal than previously reported fractures. Second, our pre-placed BP can guide an accurate reaming and subsequent tight bone-nail construct $[20,25,26]$. Third, modern perspective technique allows sufficient visualization. The nail tip can be accurately inserted into the hardest area of the subchondral bone and the distal interlocking screws can be accurately locked to avoid the "wiper effect" (Fig. 1-g) [27].

In the BS group, it requires an additional intraoperative procedure to replace the temporary Steinmann pins with screws, but in the BP group, the temporary Steinmann pins can be pulled out directly. This explains why the operating time was significantly shorter in the BP group $[15,16,28]$. The Steinmann pin was used during reduction in both groups, and no intraoperative adverse event occured. After surgery, two patients underwent removal of the permanent BS due to soft tissue irritation, but this is not a significant difference $(P=0.139)$. Of note, one patient in the BP group underwent a 4-degree loss of reduction because the bone around the distal interlocking screws was destroyed during drilling. For such patients, the addition of a permanent BS may get better results.

The major limitation of our study was its nonrandomized design. This allowed selection bias to obscure results as surgeons likely treated more difficult fractures with permanent BSs. Therefore, prospective studies with large sample size are needed to confirm the efficiency of temporary BPs. Another limitation is that we only evaluated acute, closed fractures of the distal tibia shaft, which were more stable and easier to heal. Open fractures and OTA 43 fractures should be an area of future research.

\section{Conclusion}


For acute distal tibia shaft fractures, the modern IMN fixation alone can provide sufficient stability to maintain the reduction during healing. Both temporary BP and permanent BS can help achieve equivalent overall alignment, but the BP technique is simpler and takes less time.

\section{Abbreviations}

IMN: Intramedullary nailing; BS: blocking screw; BP: blocking pin

\section{Declarations}

\section{Ethics approval and consent to participate}

This study was approved by the Institutional Review Board (IRB) of the 3rd Hospital of Hebei Medical University, Shijiazhuang, PR China. All patients participating in this study signed the written informed consent.

\section{Consent for publication}

Written informed consent was obtained from each patients' parents for the publication of this study and the accompanying images.

\section{Availability of data and materials}

The datasets generated and/or analyzed during the current study are not in public but are available from the corresponding author on reasonable request.

\section{Competing interests}

The authors declare that they have no competing interests

\section{Funding support}

This study was supported by Talent training plan of hebei province in 2013(A2013003043). The funders had no effect on the study design, data collection and publication of the manuscript.

\section{Authors' contributions}

HTW designed and supervised the study; LJM and SQY searched relevant studies on the subject; SL and PCR analyzed data; XDB and ZHS wrote the manuscript and PCW made manuscript revisions. XDB 


\section{Acknowledgements}

Not applicable.

\section{References}

1. Larsen P, Elsoe R, Hansen SH, Graven-Nielsen T, Laessoe U, Rasmussen S: Incidence and epidemiology of tibial shaft fractures. Injury 2015, 46(4):746-750.

2. Attal R, Hansen M, Kirjavainen M, Bail H, Hammer TO, Rosenberger R, Hontzsch D, Rommens PM: A multicentre case series of tibia fractures treated with the Expert Tibia Nail (ETN). Arch Orthop Trauma Surg 2012, 132(7):975-984.

3. Triantafillou K, Barcak E, Villarreal A, Collinge C, Perez E: Proper Distal Placement of Tibial Nail Improves Rate of Malalignment for Distal Tibia Fractures. Journal of orthopaedic trauma 2017, 31(12):e407-e411.

4. Hu L, Xiong Y, Mi B, Panayi AC, Zhou W, Liu Y, Liu J, Xue H, Yan C, Abududilibaier A et al: Comparison of intramedullary nailing and plate fixation in distal tibial fractures with metaphyseal damage: a meta-analysis of randomized controlled trials. Journal of orthopaedic surgery and research 2019, 14(1):30.

5. Vallier HA, Le TT, Bedi A: Radiographic and clinical comparisons of distal tibia shaft fractures (4 to $11 \mathrm{~cm}$ proximal to the plafond): plating versus intramedullary nailing. Journal of orthopaedic trauma 2008, 22(5):307-311.

6. Li Y, Jiang X, Guo Q, Zhu L, Ye T, Chen A: Treatment of distal tibial shaft fractures by three different surgical methods: a randomized, prospective study. International orthopaedics 2014, 38(6):12611267.

7. Vallier HA, Cureton BA, Patterson BM: Randomized, prospective comparison of plate versus intramedullary nail fixation for distal tibia shaft fractures. Journal of orthopaedic trauma 2011, 25(12):736-741.

8. Krettek C, Miclau T, Schandelmaier P, Stephan C, Mohlmann U, Tscherne H: The mechanical effect of blocking screws ("Poller screws") in stabilizing tibia fractures with short proximal or distal fragments after insertion of small-diameter intramedullary nails. Journal of orthopaedic trauma 1999, 13(8):550-553.

9. Krettek C, Stephan C, Schandelmaier P, Richter M, Pape HC, Miclau T: The use of Poller screws as blocking screws in stabilising tibial fractures treated with small diameter intramedullary nails. The Journal of bone and joint surgery British volume 1999, 81(6):963-968.

10. Ricci WM, O'Boyle M, Borrelli J, Bellabarba C, Sanders R: Fractures of the proximal third of the tibial shaft treated with intramedullary nails and blocking screws. Journal of orthopaedic trauma 2001, 
15(4):264-270.

11. Moongilpatti Sengodan M, Vaidyanathan S, Karunanandaganapathy S, Subbiah Subramanian S, Rajamani SG: Distal tibial metaphyseal fractures: does blocking screw extend the indication of intramedullary nailing? ISRN Orthop 2014, 2014:542623.

12. Shahulhameed A, Roberts CS, Ojike NI: Technique for precise placement of poller screws with intramedullary nailing of metaphyseal fractures of the femur and the tibia. Injury 2011, 42(2):136139.

13. Stedtfeld HW, Mittlmeier T, Landgraf P, Ewert A: The logic and clinical applications of blocking screws. The Journal of bone and joint surgery American volume 2004, 86-A Suppl 2:17-25.

14. Guerado $E$, Bertrand ML: Malalignment in intramedullary nailing. How to achieve and to maintain correct reduction? Injury 2017, 48 Suppl 1:S30-S34.

15. Wang Q, Luo JN, Li XB: Kirschner wire as blocking screws for the treatment of tibial metaphyseal fractures. Zhongguo gu shang = China journal of orthopaedics and traumatology 2014, 27(12):10401042.

16. Poyanli OS, Soylemez MS, Ozkut AT, Esenkaya I, Unal OK, Kilincoglu V: GUse of provisional K wires instead of Poller screws for treatment of diametaphyseal fractures of the distal femur and proximal and distal tibia. Acta orthopaedica Belgica 2016, 82(3):579-585.

17. Hannah A, Aboelmagd T, Yip G, Hull P: A novel technique for accurate Poller (blocking) screw placement. Injury 2014, 45(6):1011-1014.

18. Brinkmann E, DiSilvio F, Tripp M, Bernstein M, Summers H, Lack WD: Distal Nail Target and Alignment of Distal Tibia Fractures. Journal of orthopaedic trauma 2019, 33(3):137-142.

19. Freedman EL, Johnson EE: Radiographic analysis of tibial fracture malalignment following intramedullary nailing. Clin Orthop Relat Res 1995(315):25-33.

20. Avilucea FR, Triantafillou K, Whiting PS, Perez EA, Mir HR: Suprapatellar Intramedullary Nail Technique Lowers Rate of Malalignment of Distal Tibia Fractures. Journal of orthopaedic trauma 2016, 30(10):557-560.

21. Chan DS, Nayak AN, Blaisdell G, James CR, Denard A, Miles J, Santoni BG: Effect of distal interlocking screw number and position after intramedullary nailing of distal tibial fractures: a biomechanical study simulating immediate weight-bearing. Journal of orthopaedic trauma 2015 , 29(2):98-104.

22. De Giacomo AF, Tornetta P, 3rd: Alignment After Intramedullary Nailing of Distal Tibia Fractures Without Fibula Fixation. Journal of orthopaedic trauma 2016, 30(10):561-567.

23. Gao KD, Huang JH, Li F, Wang QG, Li HQ, Tao J, Wang JD, Wu XM, Wu XF, Zhou ZH et al: Treatment of aseptic diaphyseal nonunion of the lower extremities with exchange intramedullary nailing and blocking screws without open bone graft. Orthopaedic surgery 2009, 1(4):264-268.

24. Goldzak M, Biber R, Falis M: Optimal use of transmedullary support screws and fibular management in distal tibial fracture nailing based on a new biomechanical classification. Injury 2019, 50 Suppl 3:17-22. 
25. Obremskey WT, Medina M: Comparison of intramedullary nailing of distal third tibial shaft fractures: before and after traumatologists. Orthopedics 2004, 27(11):1180-1184.

26. Stinner DJ, Mir H: Techniques for intramedullary nailing of proximal tibia fractures. The Orthopedic clinics of North America 2014, 45(1):33-45.

27. Talerico M, Ahn J: Intramedullary Nail Fixation of Distal Tibia Fractures: Tips and Tricks. Journal of orthopaedic trauma 2016, 30 Suppl 4:S7-S11.

28. Biewener A, Grass R, Holch M, Zwipp H: [Intramedullary nail placement with percutaneous Kirschner wires. Illustration of method and clinical examples]. Der Unfallchirurg 2002, 105(1):65-70.

\section{Figures}
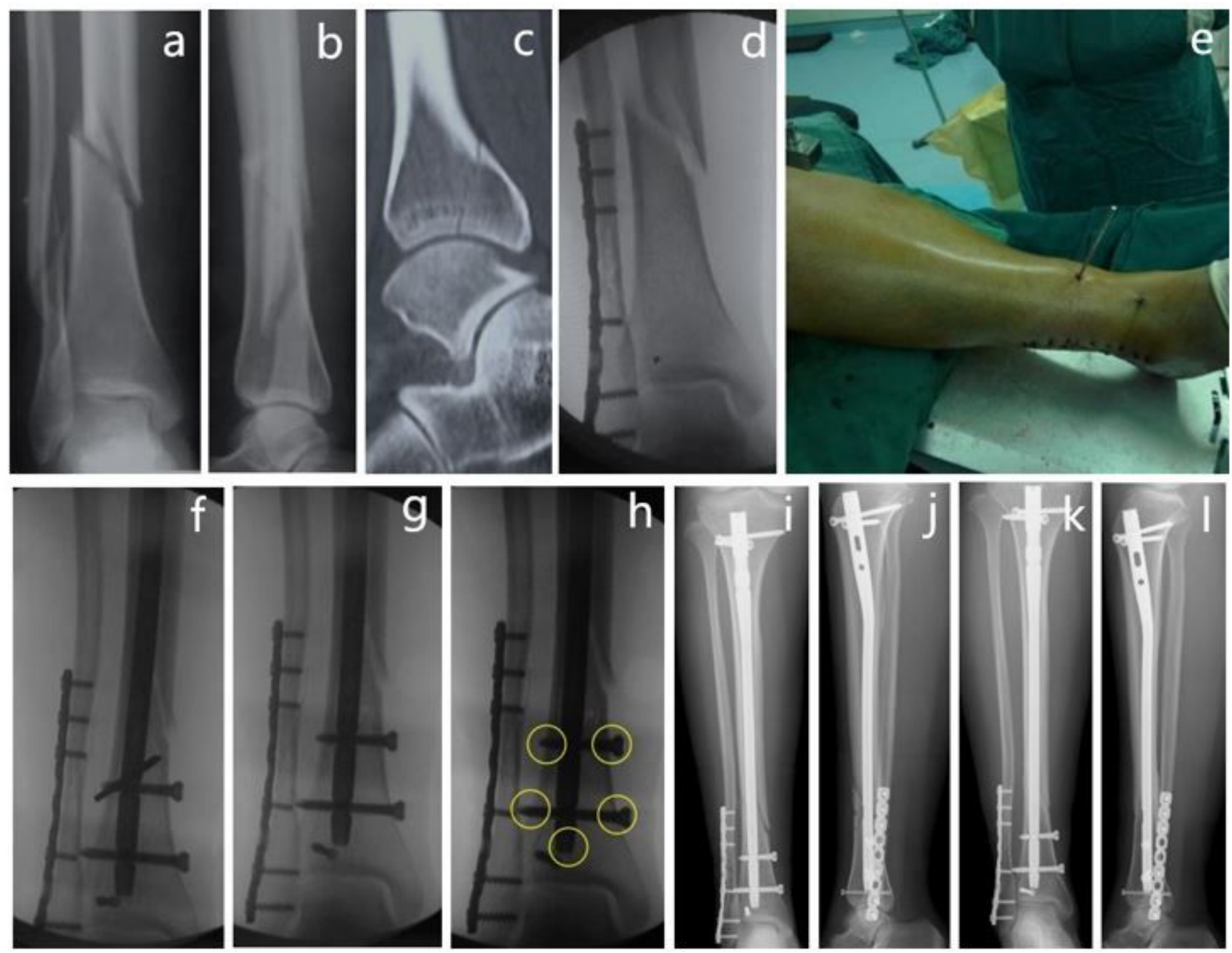

Figure 1 
Preoperative, intraoperative, and postoperative graphs of a patient with a distal tibia shaft fracture a-c Preoperative X-ray and CT $d$ The concomitant distal fibula and posterior malleolar fractures were fixed e-f A blocking pin was introduced to facilitate fracture reduction g-h The blocking pin was removed after the nail was locked $\mathrm{i}-\mathrm{j}$ Postoperative X-ray $\mathrm{k}-\mathrm{I}$ The fracture healed at 17 weeks after surgery 